\author{
International Journal of Contemporary Mathematical Sciences \\ Vol. 9, 2014, no. 14, 677 - 683 \\ HIKARI Ltd, www.m-hikari.com \\ http://dx.doi.org/10.12988/ijcms.2014.4543
}

\title{
A Synchronization Criterion for a Class of Sinusoidal Chaotic Maps via Linear Controller
}

\author{
Adel Ouannas \\ LAMIS Laboratory, Department of Mathematics and Computer Science \\ University of Tebessa, Algeria \\ Copyright (c) 2014 Adel Ouannas. This is an open access article distributed under the \\ Creative Commons Attribution License, which permits unrestricted use, distribution, and \\ reproduction in any medium, provided the original work is properly cited.

\begin{abstract}
In this paper, the problem of synchronization of some class of chaotic systems in discrete-time is investegated. Based on Lyapunov stability theory and new linear control method, a new criterion for chaos synchronization in 2-D discrete sinusoidal maps is derived. Numerical simulation validate the main result of this paper.
\end{abstract}

Keywords: Synchronization criterion, sinusoidal chaotic maps, Lyapunov stability, linear controllers

\section{Introduction}

Synchronization in chaotic systems has been received a great deal of attention among scientists from various research fields, due to the variety of important applications $[1,2,3]$. Many types of synchronization have been presented $[4,5,6]$ and various methods and techniques have been developed for chaos synchronization $[7,8]$, their main attention was focused on the synchronization in continuous-time chaotic systems. However, many models mathematics of physical processes, chemical processes and biological processes are given by discrete chaotic dynamical systems. For this reason there are demand to develop methods, and effective techniques for chaos synchronization in discrete-time. Recently, more and more attention has been paid to the synchronization of chaos in discrete-time dynamical systems [9, 10], due it's applications in secure communication $[11,12]$. 
The main aim of this paper, is to develop a simple criterion for the synchronization of two coupled general chaotic systems given by sinusoidal terms in discrete-time. Using Lyapunov stability theory and new synchronization controllers given in linear form, a new criterion for discrete chaos synchronization is derived.

This paper is organized as follows. In section 2, the drive-response chaotic systems addressed in this paper are provided. In section 3, our synchronization control mathod is introduced and a new chaos synchronization criterion is derived. In section 4, the proposed criterion is applied to achieve global synchronization between two identical modified Hénon maps [13]. In section 5 , conclusion is given.

\section{Identical drive-response chaotic maps}

We consider the drive and the response chaotic systems are in the following forms

$$
\begin{aligned}
& x_{i}(k+1)=\sum_{j=1}^{2} a_{i j} x_{j}(k)+\sum_{j=1}^{2}\left(\alpha_{i j} \cos x_{j}(k)+\beta_{i j} \sin x_{j}(k)\right)+\gamma_{i}, \quad i=1,2 . \quad(1) \\
& y_{i}(k+1)=\sum_{j=1}^{2} a_{i j} y_{j}(k)+\sum_{j=1}^{2}\left(\alpha_{i j} \cos y_{j}(k)+\beta_{i j} \sin y_{j}(k)\right)+\gamma_{i}+u_{i}, \quad i=1,(22)
\end{aligned}
$$

where $X(k)=\left(x_{1}(k), x_{2}(k)\right)^{T}, Y(k)=\left(y_{1}(k), y_{2}(k)\right)^{T}$ are the state vectors of the drive and the response systems,respectively, $\left(a_{i j}\right) \in \mathbb{R}^{2 \times 2},\left(\alpha_{i j}\right) \in \mathbb{R}^{2 \times 2}$, $\left(\beta_{i j}\right) \in \mathbb{R}^{2 \times 2},\left(\gamma_{i}\right)_{1 \leq i \leq 2}$ are real numbers and $u_{i},(i=1,2)$, are controllers to be determined.

The synchronization errors are defined as

$$
e_{i}(k)=y_{i}(k)-x_{i}(k), \quad(i=1,2),
$$

then the synchronization problem is to find $u_{i},(i=1,2)$, which stabilize the synchronization errors (3), then the aim of synchronization is to make $\lim _{k \longrightarrow+\infty} e_{i}(k)=$ $0, \quad(i=1,2)$.

\section{Synchronization Criterion}

The synchronization errors between the drive system (1) and the response system (2), can be derived as

$$
e_{i}(k+1)=\sum_{j=1}^{2} b_{i j} e_{j}+R_{i}+u_{i}, \quad i=1,2
$$


where

$R_{i}=\sum_{j=1}^{2} \gamma_{i j}\left(\cos y_{j}(k)-\cos x_{j}(k)\right)+\sum_{j=1}^{2} \delta_{i j}\left(\sin y_{j}(k)-\sin x_{j}(k)\right), \quad i=1,2$.

Now, we have the following result.

Theorem 1 The drive system (1) and the response system (2) are globally synchronized under the following controllers

$$
u_{i}=-\sum_{j=1}^{2} l_{i j} e_{j}, \quad i=1,2
$$

if the control canstants $\left(l_{i j}\right)_{1 \leq i, j \leq 2}$ are chosen such that

$$
\left\{\begin{array}{c}
l_{i j}=b_{i j}+\left|\gamma_{i j}\right|+\left|\beta_{i j}\right|, \quad i \neq j, \quad i, \quad j=1,2 . \\
\text { and } \\
0<b_{i i}+\left|\gamma_{i i}\right|+\left|\delta_{i i}\right|-l_{i i}<1, \quad i=1,2
\end{array}\right.
$$

Proof. By substituting the control law (6) into Eq. (4), the synchronization errors can be written

$$
e_{i}(k+1)=\sum_{j=1}^{2}\left(b_{i j}-l_{i j}\right) e_{j}(k)+R_{i}, \quad i=1,2 .
$$

By using the mean value theorem, we can obtain the following formulas:

$$
\sum_{j=1}^{2} \gamma_{i j}\left(\cos y_{j}(k)-\cos x_{j}(k)\right)=\sum_{j=1}^{2} \gamma_{i j} c_{i j} e_{j}(k)
$$

and

$$
\sum_{j=1}^{2} \delta_{i j}\left(\sin y_{j}(k)-\sin x_{j}(k)\right)=\sum_{j=1}^{2} \delta_{i j} s_{i j} e_{j}(k),
$$

where $\left|c_{i j}\right|<1$ and $\left|s_{i j}\right|<1,(1 \leq i, j \leq 2)$.

Substituting Eqs. (9) and (10) into Eq. (8), one can get the following formula for synchronization errors:

$$
e_{i}(k+1)=\sum_{j=1}^{2}\left(b_{i j}-l_{i j}+\gamma_{i j} c_{i j}+\delta_{i j} s_{i j}\right) e_{j}(k), \quad i=1,2
$$


Now, to prove the zero stability of synchronization errors (11), we consider the following quadratic Lyapunov function

$$
V(e(k))=\sum_{i=1}^{2} e_{i}^{2}(k),
$$

then, we obtain

$$
\begin{aligned}
\Delta V(e(k)) & =\sum_{i=1}^{2}\left[e_{i}^{2}(k+1)-e_{i}^{2}(k)\right] \\
& =\sum_{i=1}^{2}\left[\left(\sum_{j=1}^{2}\left(b_{i j}+\gamma_{i j} c_{i j}+\delta_{i j} s_{i j}-l_{i j}\right) e_{j}\right)^{2}-e_{i}^{2}(k)\right],
\end{aligned}
$$

and by using (7), we get

$$
\begin{aligned}
\Delta V(e(k)) & =\sum_{i=1}^{2}\left[\left(b_{i i}-l_{i i}+\gamma_{i i} c_{i i}+\beta_{i i} s_{i i}\right)^{2}-1\right] e_{i}^{2}(k) \\
& <\sum_{i=1}^{2}\left(\left(b_{i i}+\left|\gamma_{i i}\right|\left|c_{i i}\right|+\left|\delta_{i i}\right|\left|s_{i i}\right|-l_{i i}\right)^{2}-1\right) e_{i}^{2}(k) \\
& <\sum_{i=1}^{2}\left(\left(b_{i i}+\left|\gamma_{i i}\right|+\left|\delta_{i i}\right|-l_{i i}\right)^{2}-1\right) e_{i}^{2}(k)<0 .
\end{aligned}
$$

Thus, by Lyapunov stability it is immediate that $\lim _{k \rightarrow \infty} e_{i}(k)=0,(i=1,2)$, we conclude that the two systems (1) and (2) are globally synchronized.

Remark 2 We can extend our approach to different drive-response chaotic maps but in this case the controller is taken in sinusoidal form.

\section{Numerical Example}

In this section, to observe the efficacy of our proposed method, we consider two identical sinusoidal discrete chaotic maps. In Ref. [13], Zeraoulia and Sprott proposed a new modified Hénon map

$$
\left\{\begin{array}{c}
x_{1}(k+1)=1-a \sin x_{1}(k)+b x_{2}(k), \\
x_{2}(k+1)=x_{1}(k),
\end{array}\right.
$$

where the quadratic term $x^{2}$ in the Hénon map is replaced by the nonlinear term $\sin x$. Chaotic attractors of the modified Hénon map (13) are shown in Fig. 1.

The modified Hénon map (13) is considered as the drive system, as the response system we consider the following controlled modified Hénon map

$$
\left\{\begin{array}{c}
y_{1}(k+1)=1-a \sin y_{1}(k)+b y_{2}(k)+u_{1}, \\
y_{2}(k+1)=y_{1}(k)+u_{2},
\end{array}\right.
$$

where $\left(u_{1}, u_{2}\right)^{T}$ is the controller. 


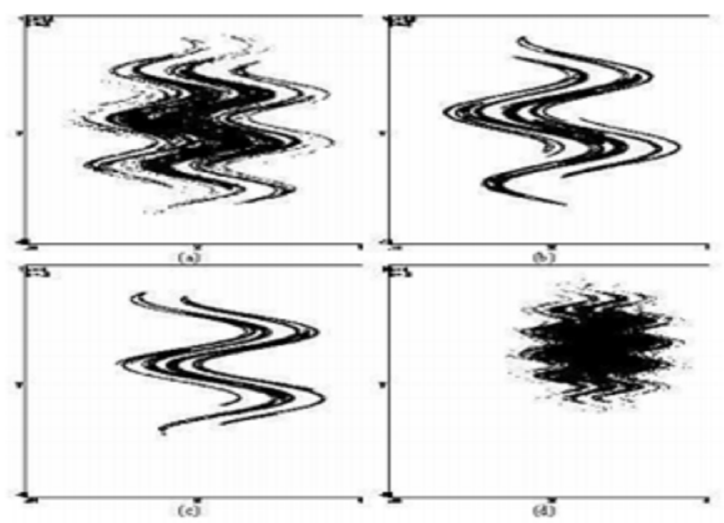

Figure 1: Chaotic multifold attractors of the map (3) obtained for (a) a $=3.4$, $\mathrm{b}=-0.8$. (b) $\mathrm{a}=3.6, \mathrm{~b}=-0.8$. (c) $\mathrm{a}=4, \mathrm{~b}=0.5$. (d) $\mathrm{a}=4, \mathrm{~b}=0.9$

Theorem 3 If $\left(l_{i}\right)_{1 \leq i \leq 2}$ are chosen such that: $-1-a<l_{1}<-a+1$ and $-1<$ $l_{2}<1$. Then, the drive system (13) and the response system (14) are globally synchronized under the synchronization controllers: $u_{1}=l_{1} e_{1}(k)-b e_{2}(k)$ and $u_{2}=-e_{1}(k)+l_{2} e_{2}(k)$.

By using Matlab, we get the numeric result that is showed in Fig.2.

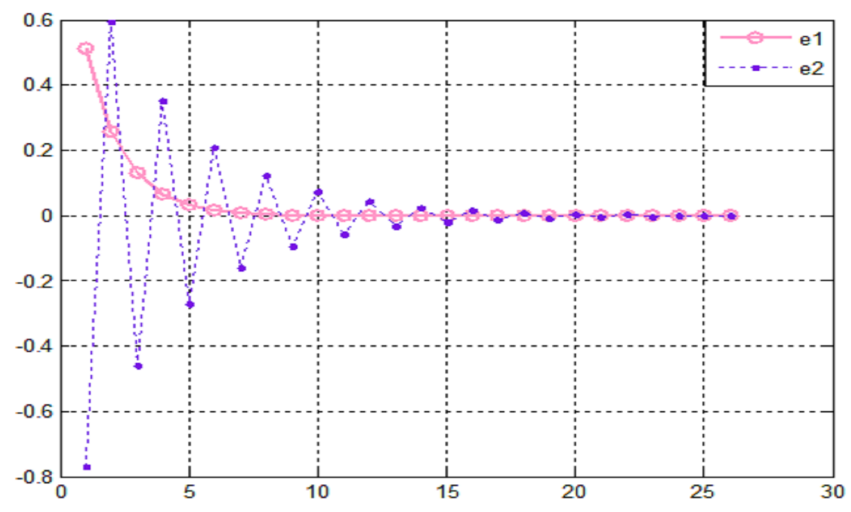

Figure 2: Time evolution of synchronization errors between the drive system (13) and the response system (14) 


\section{Conclusion}

In this paper, using Lyapunov stability theory and new controllers, a new synchronization criterion was derived for some class of sinusoidal discrete chaotic maps in 2D. Numerical example was provided to illustrate the effectiveness of the proposed method.

\section{References}

[1] L. Kocarev and U. Parlitz. General approach for chaotic synchronization with applications to communication, Phys Rev Lett, 74(1995), 5028-5037. http://dx.doi.org/10.1103/physrevlett.74.5028

[2] G S Duane, P J Webster, and J B Weiss. Go-occurrence of northern and southern hemisphere blocks as partially synchronized chaos, Journal of Atmospheric sciences, 56 (1999) 4183-4205.http://dx.doi.org/10.1175/15200469(1999)056¡4183:coonas ¿2.0.co;2

[3] B. Blasius and L. Stone, Chaos and phase synchronization in ecological systems, International Journal of Bifurcation and Chaos, 10 (2000) 23612380. http://dx.doi.org/10.1142/s0218127400001511

[4] Y.W. Wang and Z.H. Guan, Generalized synchronization of continuous chaotic systems, Chaos Solition Fract, 27(2006), 97101.http://dx.doi.org/10.1016/j.chaos.2004.12.038

[5] X. Zhang and H. Zhu. Anti-synchronization of two different hyperchaotic systems via active and adaptive control, Inter J Nonli Sci, 6(2008), 216223.

[6] J. Qiang. Projective synchronization of a new hyperchaotic Lorenz system chaotic systems, Phys Lett A, 370(2007), 40-45. http://dx.doi.org/10.1016/j.physleta.2007.05.028

[7] J. Zhao and J.Lu. Parameter identification and backstepping control of uncertain Lu system, Chaos Solition Fract, 17(2003), 721-729.

[8] H.T. Yau, Design of adaptive sliding mode controller for chaos synchronization with uncertainties, Chaos Solition Fract, 22(2004), 341-347. http://dx.doi.org/10.1016/j.chaos.2004.02.004

[9] A. Ouannas. A new Q-S synchronization scheme for discrete chaotic systems. Far East Journal of Applied Mathematics, 84 (2) 89-94. 
[10] A. Ouannas. On Full-State Hybrid Projective Synchronization of General Discrete Chaotic Systems. Journal of Nonlinear Dynamics, volume 2014. http://dx.doi.org/10.1155/2014/983293

[11] J.G. Lu and Y.G. Xi. Chaos communication based on synchronization of discrete-time chaotic systems. Chin. Phys. 14(2)(2005), 274-278. http://dx.doi.org/10.1088/1009-1963/14/2/010

[12] E. Solak. Cryptanalysis of observer based discrete-time chaotic encryption schemes. Inter. J. Bifur. Chaos. 15(2), (2005), 653-658. http://dx.doi.org/10.1142/s0218127405012260

[13] E. Zeraoulia, J. C. Sprott. A Two-dimensional Discrete Mapping with $C^{\infty}$ Multifold Chaotic Attractors. Electronic Journal of Theoretical Physics 5, No. 17 (2008) 107-120.

Received: May 11, 2014; Published: November 21, 2014 